\title{
Incremental clinical and economic impact of recombinant zoster vaccination: real-world data in a budget impact model
}

Brandon J Patterson, PharmD, PhD; William L Herring, PhD; Desiree Van Oorschot, MSc; Desmond Curran, PhD; Justin Carrico, BS; Yuanhui Zhang, PhD; Bradley K Ackerson, MD; Katia Bruxvoort, MPH, PhD; Lina S Sy, MPH; and Hung-Fu Tseng, MPH, PhD

\section{What is already known about this subject}

- In 2017, the adjuvanted recombinant zoster vaccine (RZV) was approved by the FDA for herpes zoster $(\mathrm{HZ})$ prevention in immunocompetent adults aged 50 years and older, joining the live zoster vaccine (ZVL) as U.S.-marketed vaccines against $\mathrm{HZ}$.

- The Advisory Committee on Immunization Practices preferentially recommended $R Z V$ for the prevention of $\mathrm{HZ}$ and related complications, including vaccination of adults who had previously received ZVL.

- Assessing the incremental clinical and economic impact of increasing $\mathrm{HZ}$ vaccination coverage is essential for population-based decision making; however, the heterogeneity across populations covered by health plans dictates the need for plan-specific data for decision making.

\section{ABSTRACT}

BACKGROUND: In 2017, the FDA approved the adjuvanted recombinant zoster vaccine (RZV) for the prevention of herpes zoster $(\mathrm{HZ})$ in immunocompetent adults aged 50 years and older. RZV joined zoster vaccine live (ZVL) as U.S.-marketed vaccines against $\mathrm{HZ}$. The Advisory Committee on Immunization Practices preferentially recommended use of RZV over ZVL. In order to inform populationbased decision makers (PBDMs) about the incremental clinical and economic impact

\section{What this study adds}

- This study estimates the budget impact and likely clinical outcomes as a result of adopting RZV for $\mathrm{HZ}$ prevention compared with continuing with ZVL for hypothetical U.S. national health plans, and for a large, integrated delivery network (IDN).

- Over time, the incremental permember-per-month (PMPM) budget impact reduced as the shorter-term costs of adopting the new vaccine technology were increasingly outweighed by the longer-term benefits of vaccination via $\mathrm{HZ}$ case avoidance.

- The actual PMPM budget impact was dependent upon specific plan population attributes (age distribution of the population, cost sharing, vaccine coverage, epidemiology within health plan population); this can be determined independently by other IDNs as the model used in this study is being made publicly available.

of RZV adoption, budget impact (BI) models may be used. Populating such models with national data can inform PBDMs about the incremental value of RZV adoption nationally; however, heterogeneity across health plans requires the inclusion of plan-specific data to ensure the relevance of modeling outcomes for plan-specific decision makers.

OBJECTIVE: To investigate the clinical and economic outcomes associated with the adoption of RZV in nationally representative populations with commercial and Medicare

\section{Author affiliations}

Brandon J Patterson, PharmD, PhD; GSK, Philadelphia, PA. Desiree Van Oorschot, MSc, and Desmond Curran, PhD, GSK, Wavre, Belgium. William L Herring, PhD; Justin Carrico, BS; and Yuanhui Zhang, PhD, RTI Health Solutions, Research Triangle, NC. Bradley K Ackerson, MD; Katia Bruxvoort, $\mathrm{MPH}, \mathrm{PhD}$; Lina S Sy, MPH; and Hung-Fu Tseng, MPH, PhD, Kaiser Permanente Southern California, Pasadena.

AUTHOR CORRESPONDENCE: Brandon J Patterson, 215.983.4608, brandon.j.patterson@gsk.com coverage and to demonstrate the effect of the heterogeneity of health plans using realworld data from a large, integrated delivery network (IDN).

METHODS: We used a publicly available BI model. The model accounts for national and IDN-collected population characteristics (size, age distribution) and epidemiological data (incidence of $\mathrm{HZ}$ and complications, $\mathrm{HZ}$ recurrence rate), vaccine characteristics from randomized controlled trials and observational studies (efficacy, waning, second dose 
compliance for RZV, adverse event rate), national costs (vaccine, direct medical for $\mathrm{HZ}$, complications, and vaccine adverse events), and current and anticipated vaccine coverage. We assessed incremental clinical ( $\mathrm{HZ}$ cases and complications) and economic (permember-per-month [PMPM] costs) impact at 5-year to 15-year time horizons, comparing scenarios where RZV is solely implemented with one where only ZVL is utilized.

RESULTS: Following the adoption of RZV, the incremental HZ cases avoided over 5 and 15 years were estimated to be 1,800 and 15,000 for a commercial plan, 3,800 and 21,000 for a Medicare plan, and 8,600 and 71,000 for a specific IDN. The incremental PMPM budget impact over the same time horizons was estimated to be $\$ 0.42$ and $\$ 0.31$, respectively, for a commercial plan, $\$ 0.35$ and $\$ 0.10$ for a Medicare plan, and $\$ 0.39$ and $\$ 0.25$ for a specific IDN. The differences in results across plans resulted from the population age distribution, the vaccine copay (applied in the Medicare scenario only), the vaccine coverage in the plan, and other plan-specific factors affecting disease epidemiology and costs per case of $\mathrm{HZ}$.

CONCLUSIONS: Model projections indicated that RZV adoption avoided $\mathrm{HZ}$ cases and related complications, with the PMPM budget impact dependent on plan-specific factors. As health gains increased over time, the incremental costs incurred were found to decrease as the shorter-term costs of adopting the new vaccine were increasingly offset by the longer-term benefits of vaccination.

Herpes zoster (HZ) is an infection caused by reactivation of the varicella zoster virus that occurs in aging people as specific cellular immunity against the virus declines. ${ }^{1}$ In addition to presenting as an acute, painful rash, the infection may lead to pain persisting 90 days or more (defined as postherpetic neuralgia [PHN]); furthermore, it can cause other serious complications such as HZ ophthalmicus (eye complications including keratitis, uveitis/iritis, conjunctivitis, and loss of vision). ${ }^{2}$

$\mathrm{HZ}$ and its associated complications present a substantial economic burden to health systems, with estimates ranging from $\$ 700$ million (2015) to $\$ 1.9$ billion (2013) in annual medical costs for the U.S. population. ${ }^{3,4}$ This burden is projected to increase over time as the U.S. population ages. ${ }^{5}$

There are 2 vaccines approved by the U.S. Food and Drug Administration (FDA) for HZ prevention. In 2008, the Advisory Committee on Immunization Practices (ACIP) recommended vaccination of adults aged 60 years and older. ${ }^{6}$ In 2006, the zoster vaccine live (ZVL; Zostavax, Merck \& Co.) was the first vaccine approved for the prevention of $\mathrm{HZ}$ in individuals aged 50 years and older, given as a single dose, ${ }^{6,7}$ but its protection wanes significantly over time (from $69 \%$ down to $4 \%$ over 8 years in adults aged 60 years and older). ${ }^{8,9}$ In 2017, the adjuvanted recombinant zoster vaccine
(RZV; Shingrix, GSK) was approved by the FDA for HZ prevention in adults aged 50 years and older, given in a 2-dose sequence. ${ }^{7} \mathrm{RZV}$, with an efficacy of $>90 \%$ in individuals aged 50 years and older and minimal waning over 4 years,,$^{10,11}$ received a preferential recommendation from the ACIP for $\mathrm{HZ}$ prevention among all immunocompetent adults aged 50 years and older, including a recommendation for RZV vaccination in adults previously vaccinated with ZVL. ${ }^{9}$

Assessing the incremental clinical and economic impact of adopting a novel health technology is critical for population-based decision makers (PBDMs). Health care expenditures are of particular increasing concern to public and private PBDMs as the U.S. population continues to age. ${ }^{12}$ In 2016, the Journal of Managed Care + Specialty Pharmacy published a budget impact analysis relating to the implementation of ZVL from a health maintenance organization perspective. ${ }^{13}$ The model provided information to PBDMs about the incremental value of ZVL adoption and the annual effect on health plan costs related to HZ prevention and management of $\mathrm{HZ}$ and its associated complications. Since this publication, the FDA approved RZV, more data became available on the waning efficacy of ZVL, ${ }^{9}$ and the ACIP preferentially recommended the use of the newer vaccine.

While a budget impact (BI) model populated with national data can inform PBDMs about the incremental value of HZ vaccination in nationally representative populations, heterogeneity across health plans highlights the need for plan-specific data to enhance the relevance of model outcomes for plan-specific decision makers. This study (GSK study identifier: HO-17-18378) was therefore conducted to project clinical and economic outcomes associated with the adoption of RZV for hypothetical U.S. national health plans and for a large, integrated delivery network (IDN).

\section{Methods}

\section{MODELING APPROACH}

Using Microsoft Excel (Microsoft, Redmond, WA), an interactive BI model was developed to project the impact to a health plan's budget resulting from the introduction and uptake of RZV. The model considered the U.S. population eligible for vaccination against $\mathrm{HZ}$, people aged 50 years and older who were classified into 7 age groups: $50-54$ years, $55-$ 59 years, $60-64$ years, $65-69$ years, $70-74$ years, $75-79$ years, and 80 years and older. The size of a health plan's population of members under the age of 50 years (classified into 2 age groups: $0-17$ years and 18-49 years) was considered for the purposes of calculating the incremental per-memberper-month (PMPM) plan costs resulting from the vaccination program change and was assumed to remain constant over the model time horizon. As the population aged in the 
second and all subsequent years of the time horizon, a new cohort of individuals aged 50 years was added to the population. The size of this new cohort was assumed to be one fifth of the starting size of the youngest eligible 5-year age group. All-cause mortality rates (\%) in the United States for 5-year age groups were derived from the Centers for Disease Control and Prevention (CDC) Life Tables. ${ }^{14}$ These rates for ages $0-49$ years are not reported, as these ages were not explicitly modeled. HZ-related mortality was not considered, as the rate of death among HZ cases is low, and $\mathrm{HZ}$ is rarely the underlying cause of death. ${ }^{15}$

The annual rates of initial and recurrent cases of $\mathrm{HZ}$ and rates of associated PHN and nonpain complications (i.e., ocular, neurological, cutaneous, and other nonpain complications) were considered in the model; the default values and assumptions for these epidemiological inputs have been described previously. ${ }^{16,17}$

Vaccine efficacy, waning of efficacy, and adverse event inputs used in this model also have been described previously. ${ }^{16,18}$ Efficacy against HZ and PHN was included for those vaccinated with RZV and ZVL, with the baseline efficacy for each vaccine varying by age and with efficacy waning based on the years since vaccination. For individuals previously vaccinated with ZVL, the starting efficacy was based on the average time since vaccination and waning of ZVL protection in that time. Incidence of vaccine-related adverse events was applied to each dose of vaccine administered.

The perspective of the model was that of a third-party payer. Vaccine acquisition cost per dose of RZV (U.S. \$144.20) was based on the September 2019 CDC Vaccine Price List (private sector cost per dose) and ZVL (U.S. \$212.67) based on the June 2018 CDC Vaccine Price List (private sector cost

\section{FIGURE 1 Cohort-Level Annual Transitions in the Budget Impact Model}

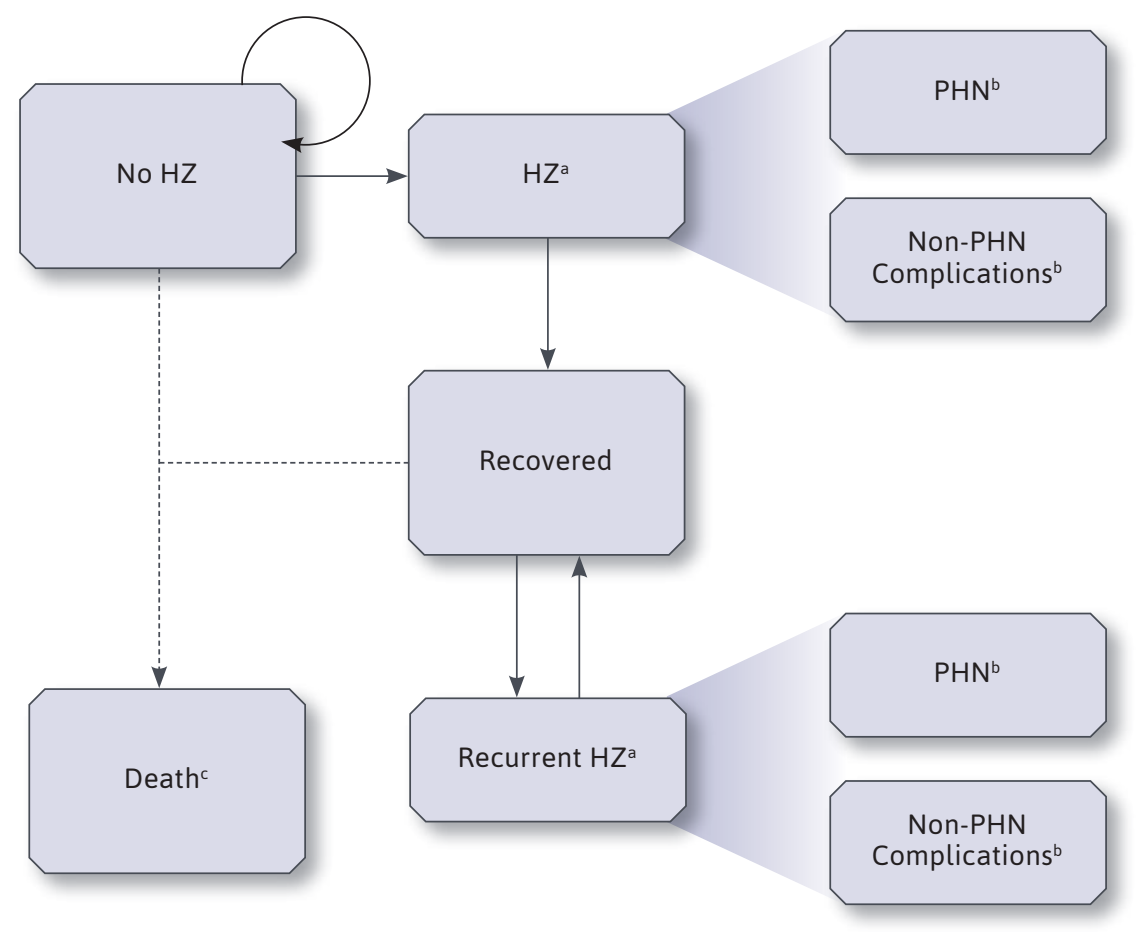

Source: Adapted from Figure 5 of the article "Assessment of the potential public health impact of Herpes Zoster vaccination in Germany" (http://dx.doi.org/10.1080/21645515.2017.1345399) by Desmond Curran, Desirée Van Oorschot, Lijoy Varghese, Lidia Oostvogels, Tomas Mrkvan, Romulo Colindres, Alfred von Krempelhuber, and Anastassia Anastassopoulou, Hum Vaccin Immunother, 2017;13(10):2213-21, available under the terms of the Creative Commons Attribution License (http://creativecommons.org/licenses/ by/4.0/). Copyright 2017 GSK

$a^{\prime \prime} \mathrm{HZ}$ " and "Recurrent $\mathrm{HZ}$ " are modeled as events occurring during years spent in the "No HZ" and

"Recovered" health states, respectively.

b"PHN" and "Non-PHN Complications" are modeled as proportions of overall "HZ" (or "Recurrent HZ") cases. "All-cause mortality can occur from either the "No HZ" or the "Recovered" health states. HZ-related mortality is not included in the model.

$\mathrm{HZ}=$ herpes zoster; $\mathrm{PHN}=$ postherpetic neuralgia

per dose) (as ZVL is not listed in the price list from July 2018 onward). ${ }^{19,20}$ The administration cost per dose of RZV or ZVL was assumed to be U.S. $\$ 20 .{ }^{21}$ Copay and coinsurance were not considered for the commercial health plans, in accordance with the Affordable Care Act preventive services requirements ${ }^{22}$; however, a copay of U.S. \$50 per vaccine dose was assumed for the Medicare plan, supported by internally collected data.

Costs due to vaccine-related adverse events varied by vaccine.
Default values, sources, and assumptions for adverse event-related and HZ-related costs have been previously described. ${ }^{16}$ All costs were inflated to 2018 U.S. dollars using the medical care component of the Consumer Price Index..$^{23}$

Health care utilization in the form of outpatient office visits and inpatient admissions per case of $\mathrm{HZ}$ was estimated based on 2008-2011 claims data from the Commercial Claims and Encounters database and the Medicare Supplemental and 


\begin{tabular}{l|c|c|c|c}
\hline \multicolumn{1}{c}{ TABLE 1 } & \multicolumn{2}{c}{$\begin{array}{c}\text { Population Characteristics and HZ Vaccine Coverage for } \\
\text { Hypothetical U.S. Commercial and Medicare Health Plans }\end{array}$} \\
\cline { 1 - 3 } $\begin{array}{l}\text { Age Group } \\
\text { (Years) }\end{array}$ & $\begin{array}{c}\text { Population Size by Age Group } \\
\text { U.S. Commercial } \\
\text { Health Plan }\end{array}$ & $\begin{array}{c}\text { U.S. Medicare } \\
\text { Health Plan }\end{array}$ & $\begin{array}{c}\text { Annual } \\
\text { All-Cause } \\
\text { Mortality Rate (\%) }\end{array}$ & $\begin{array}{c}\text { Vaccine Coverage } \\
\text { with ZVL (\%) }\end{array}$ \\
\hline All ages & $1,000,000$ & $1,000,000$ & & 0.0 \\
\hline $0-17$ & 213,155 & 34 & - & 0.0 \\
\hline $18-49$ & 450,105 & 57,558 & - & 0.0 \\
\hline $50-54$ & 78,132 & 23,688 & 0.5 & 21.7 \\
\hline $55-59$ & 76,291 & 43,524 & 0.7 & 34.2 \\
\hline $60-64$ & 66,712 & 37,561 & 1.0 & 34.2 \\
\hline $65-69$ & 38,891 & 269,623 & 1.5 & 34.2 \\
\hline $70-74$ & 27,794 & 194,808 & 2.3 & 34.2 \\
\hline $75-79$ & 19,664 & 146,940 & 3.7 & 0.0 \\
\hline $80+$ & 29,255 & 226,262 & $6.1-43.5$ & \\
\hline
\end{tabular}

$\mathrm{HZ}=$ herpes zoster; $\mathrm{ZVL}=$ zoster vaccine live.

Coordination of Benefits database. ${ }^{24}$ Resource utilization was assumed to be the same for unvaccinated and vaccinated $\mathrm{HZ}$ cases. HZ-related health care resource utilization was estimated as an independent outcome separate from HZ-related costs. In other words, the calculated numbers of outpatient office visits and inpatient admissions due to HZ were independent outcomes of the model and were not used to calculate HZ-related costs in the model, since HZ-related costs were estimated from other sources on a case basis. ${ }^{16,25}$

The model considers time horizons from 1 to 15 years. To estimate the incidence of $\mathrm{HZ}$ and its complications, cohort-level calculations were replicated for each of 5 vaccine cohorts (2-dose RZV, 1-dose RZV, ZVL, previously vaccinated with ZVL, and no vaccination) for each age range (Figure 1). Each replication tracked a cohort of individuals year by year over a period of 1 to 15 years through a number of possible health states, consistent with previous costeffectiveness analyses for RZV. ${ }^{16,17}$ The population-level calculations combined vaccine coverage levels over time with the cohort-level outcomes to estimate the total number of cases of HZ and PHN in each year of the modeling horizon. The number of $\mathrm{HZ}$ and PHN cases were estimated separately and by the age range in which the cases occurred. These estimates were then used to calculate the direct HZ-related and PHN-related costs, HZ-related resource utilization, and the number of HZ-related complications and associated costs.

The model was used to compare a scenario "Without RZV," where agespecific coverage with ZVL linearly increased over initial ZVL coverage rates by $10 \%$ over 15 years and a "With RZV" scenario where the maximum RZV coverage achieved in 15 years was $65 \%$ for all age groups. Initial ZVL coverage rates were taken from 2015 National Health Interview Survey results and demonstrated slight growth in vaccination rates in recent years. ${ }^{26}$ As ZVL was only recommended for adults aged 60 years and older, negligible coverage was seen in those aged 50-59 years. In the With RZV scenario, HZ vaccine coverage for individuals previously vaccinated against $\mathrm{HZ}$ with $\mathrm{ZVL}$ was assumed to be the same as coverage in those not previously vaccinated, as the ACIP recommends that previously vaccinated individuals also receive RZV. ${ }^{9}$ Those previously vaccinated were assumed to have received $\mathrm{ZVL}$ 5 years before the start of the model time horizon. In the Without RZV scenario, revaccination with ZVL among previously vaccinated individuals was assumed to not occur. In both scenarios, cohorts entering the youngest age group eligible for vaccination were vaccinated at the specified maximum coverage levels at model entry. While this was a simplification for modeling purposes, the underpinning logic was that health care providers would incorporate recommendations and vaccinate new cohorts upon becoming eligible while also working to catch up individuals served who were older. The proportion of people receiving the second dose of RZV was assumed to be $76 \% .^{27}$

\section{COMMERCIAL AND MEDICARE PLANS}

A hypothetical 1-million-person commercial plan was populated with representative age distribution based on a recent reporting of percentages of people with private health insurance by age group. ${ }^{28}$ Additionally, a hypothetical 1-million-person Medicare plan was populated with representative distribution based on a recent report of Medicare A/B enrollees by age group (Table 1). ${ }^{29}$

\section{KPSC CASE STUDY}

To demonstrate the customization of the model to a specific U.S. health plan, data were collected by a large IDN, namely Kaiser Permanente Southern California (KPSC), which is an integrated health care organization 


\begin{tabular}{|c|c|c|c|}
\hline TAE & $\begin{array}{l}\mathrm{P} \\
\mathrm{C} \\
\mathrm{a} \\
\mathrm{C} \\
\mathrm{K}\end{array}$ & \multicolumn{2}{|c|}{$\begin{array}{l}\text { Population } \\
\text { Characteristics } \\
\text { and HZ Vaccine } \\
\text { Coverage for } \\
\text { KPSC Health Plan }\end{array}$} \\
\hline $\begin{array}{l}\text { Age } \\
\text { Group } \\
\text { (Years) }\end{array}$ & $\begin{array}{l}\text { Population } \\
\text { Size }\end{array}$ & $\begin{array}{l}\text { Annual } \\
\text { All-Cause } \\
\text { Mortality } \\
\text { Rate (\%) }\end{array}$ & $\begin{array}{l}\text { Previous } \\
\text { HZ } \\
\text { Vaccine } \\
\text { Coverage } \\
\text { with ZVL } \\
(\%)\end{array}$ \\
\hline All ages & $4,053,865$ & & \\
\hline $0-17$ & 759,550 & - & 0.0 \\
\hline $18-49$ & $1,855,193$ & - & 0.0 \\
\hline $50-54$ & 292,397 & 0.2 & 1.0 \\
\hline $55-59$ & 289,087 & 0.4 & 2.9 \\
\hline $60-64$ & 255,330 & 0.6 & 19.1 \\
\hline $65-69$ & 221,849 & 1.0 & 35.2 \\
\hline $70-74$ & 155,347 & 1.7 & 44.0 \\
\hline $75-79$ & 100,331 & 2.9 & 44.7 \\
\hline $80+$ & 124,781 & $5.1-35.3$ & 39.5 \\
\hline \multicolumn{4}{|c|}{$\begin{array}{l}\text { Note: Mean (SD) time since ZVL vaccination was } \\
4.59(2.84) \text { years. }\end{array}$} \\
\hline \multicolumn{4}{|c|}{$\begin{array}{l}\mathrm{HZ}=\text { herpes zoster; } K P S C=\text { Kaiser Permanente } \\
\text { Southern California; } S D=\text { standard deviation; } \\
Z \mathrm{VL}=\text { zoster vaccine live. }\end{array}$} \\
\hline
\end{tabular}

that provides prepaid comprehensive health care to more than 4 million members. Algorithms were defined to determine HZ epidemiology and HZrelated health care resource utilization from electronic health records, ${ }^{15}$ and the aggregated data were aligned to the model age groups.

Population demographics, all-cause mortality rates, HZ epidemiology, HZ-related health care resource utilization, and previous $\mathrm{HZ}$ vaccine coverage data at KPSC were used as inputs to the model. These inputs included the KPSC population as of January 1, 2017, with $35.5 \%(1,439,122)$ of members aged 50 years or older. The percentage of KPSC members who had received ZVL vaccination as of January 1, 2017, was estimated by age (Table 2). No copay was considered for vaccine doses administered in this scenario. Inputs for which KPSC data were not identified remained the same as for the national population scenarios, namely vaccine attributes (efficacy, waning, adverse events), HZ- and PHN-related costs, vaccine-related adverse event costs, and projected ZVL and RZV coverage for the Without RZV and With RZV scenarios, respectively.

\section{SENSITIVITY ANALYSIS}

Sensitivity analyses were performed for the 2 national scenarios and the KPSC case study to explore the effect of alternate assumptions for key model inputs on cumulative incremental case avoidance and budget impact over 5, 10 , and 15 years.

The maximum 15-year RZV vaccination coverage was varied from $55 \%$ to $75 \%$ based on uncertainty about real-world uptake of the vaccine. The proportion of people receiving the second dose of RZV was assumed to vary from $45 \%$ to $89 \%$ to reflect uncertainty in second dose compliance with RZV and the range of published estimates for other adult vaccines. ${ }^{27,30} \mathrm{HZ}$-related costs were varied based on published confidence intervals for costs per case of $\mathrm{HZ}$ without PHN. ${ }^{25}$ We assumed $20 \%$ variations from base-case values for costs per case of HZ with PHN and costs per HZ-related complication; these ranges have been previously described. ${ }^{16}$

\section{OUTCOMES}

In each model year and cumulatively over the modeling horizon, the numbers of people vaccinated with RZV and with ZVL were calculated as well as the numbers of HZ cases and HZrelated complications. Most prevalent health care resources utilized with $\mathrm{HZ}$ episodes, including annual HZrelated primary care physician (PCP) visits and hospitalizations, were calculated. ${ }^{31}$ Total vaccine-related and HZ-related costs were calculated for each model year. Costs were reported as cumulative incremental total costs at each time horizon. PMPM costs were reported based on the number of members of all ages in the health plan population, the number of members aged 18 years and older in the health plan (i.e., the number of adults), and the number of members aged 50 years and older in the health plan (i.e., the portion of the population eligible for vaccination with RZV). Costs were not discounted, as recommended by the International Society for Pharmacoeconomics and Outcomes Research's Task Force on Good Research Practices for BI models. ${ }^{32}$ The incremental budget impact was calculated each year by comparing total annual costs, including vaccine-related and HZ-related costs, between the With RZV and the Without RZV scenarios.

This study is a modeling exercise that does not meet the definition of human subject research and for which institutional review board (IRB) approval or exemption was not required. KPSC obtained IRB approval to provide epidemiologic and health care utilization inputs for the modeling exercise.

\section{Results}

\section{BASE-CASE ANALYSIS}

For hypothetical million-member U.S. commercial and Medicare health plans, it was estimated that 310,000 and 398,000 people, respectively, would be vaccinated with RZV in the 15 years following RZV introduction. For the commercial health plan, $\mathrm{cu}^{-}$ mulative incremental avoidance of HZ cases for the With RZV scenario compared with the Without RZV scenario at time horizons of 5, 10, and 15 years was estimated to be $1,800,7,000$, and 15,000 cases, respectively $(3,800$, 12,000 , and 21,000 cases for the Medicare health plan, respectively). Additionally, 35,000 HZ-related PCP visits 


\section{TABLE 3}

\begin{tabular}{|c|c|c|c|c|c|c|}
\hline \multirow[b]{2}{*}{ Outcome (Cumulative) } & \multicolumn{3}{|c|}{ Commercial Population } & \multicolumn{3}{|c|}{ Medicare Population } \\
\hline & 5 years & 10 years & 15 years & 5 years & 10 years & 15 years \\
\hline \multicolumn{7}{|c|}{ Incremental vaccine-related costs, \$ (thousands) } \\
\hline Acquisition & 22,386 & 45,637 & 67,323 & 20,923 & 38,105 & 51,107 \\
\hline Administration & 3,280 & 6,681 & 9,848 & 5,003 & 9,098 & 12,184 \\
\hline Adverse events & 3,237 & 6,557 & 9,621 & 4,715 & 8,545 & 11,430 \\
\hline Total & 28,903 & 58,875 & 86,793 & 30,641 & 55,749 & 74,721 \\
\hline Total (PMPM—all ages) & 0.48 & 0.49 & 0.48 & 0.51 & 0.46 & 0.42 \\
\hline \multicolumn{7}{|c|}{ Incremental HZ-related costs, \$ (thousands) } \\
\hline $\mathrm{HZ}$ cases without $\mathrm{PHN}$ & $(1,840)$ & $(7,110)$ & $(15,463)$ & $(4,475)$ & $(14,390)$ & $(25,576)$ \\
\hline $\mathrm{HZ}$ cases with $\mathrm{PHN}$ & $(558)$ & $(2,309)$ & $(5,382)$ & $(1,900)$ & $(6,477)$ & $(11,750)$ \\
\hline Nonpain complications & $(1,140)$ & $(4,434)$ & $(9,785)$ & $(3,212)$ & $(10,536)$ & $(18,964)$ \\
\hline Total & $(3,538)$ & $(13,853)$ & $(30,630)$ & $(9,587)$ & $(31,403)$ & $(56,290)$ \\
\hline Total (PMPM—all ages) & $(0.06)$ & $(0.12)$ & $(0.17)$ & $(0.16)$ & $(0.26)$ & $(0.31)$ \\
\hline \multicolumn{7}{|c|}{ Incremental budget impact, \$ } \\
\hline Total (thousands) & 25,365 & 45,022 & 56,162 & 21,055 & 24,346 & 18,431 \\
\hline PMPM-all ages & 0.42 & 0.38 & 0.31 & 0.35 & 0.20 & 0.10 \\
\hline PMPM-ages 18+ & 0.54 & 0.48 & 0.40 & 0.35 & 0.20 & 0.10 \\
\hline PMPM-ages $50+$ & 1.26 & 1.11 & 0.93 & 0.37 & 0.22 & 0.11 \\
\hline
\end{tabular}

$\mathrm{HZ}=$ herpes zoster; $\mathrm{PHN}=$ postherpetic neuralgia; $\mathrm{PMPM}=$ per member per month; $\mathrm{RZV}=$ adjuvanted recombinant zoster vaccine.

and 330 HZ-related hospitalizations were estimated to be avoided incrementally over 15 years for the commercial population $(56,000 \mathrm{HZ}$-related PCP visits and 990 HZ-related hospitalizations avoided for the Medicare health plan).

As shown in Table 3, uptake of RZV resulted in estimated incremental vaccination costs of $\$ 87$ million and $\$ 75$ million over 15 years for the With RZV market scenario compared with the Without RZV market scenario for commercial and Medicare health plans, respectively. Estimated incremental HZ-related costs avoided amounted to \$31 million and \$56 million for commercial and Medicare health plans, respectively. For the commercial health plan, the estimated total incremental budget impact of With RZV compared with Without RZV increased from \$25 million over 5 years to $\$ 56$ million over 15 years, while the estimated average incremental PMPM budget impact (among all plan members) decreased from $\$ 0.42$ over 5 years to $\$ 0.31$ over 15 years. For the Medicare health plan, the estimated total incremental budget impact increased from \$21 million at 5 years to $\$ 24$ million at 10 years, then decreased to \$18 million at 15 years, while the estimated average incremental PMPM budget impact decreased from $\$ 0.35$ at 5 years to $\$ 0.10$ at 15 years.

\section{KPSC CASE STUDY}

Incorporating the KPSC demographic, epidemiologic, health care utilization, and previous ZVL coverage data, the BI model projected that 1.25 million plan members would be vaccinated with RZV over 15 years. The number of $\mathrm{HZ}$ cases after 5,10 , and 15 years from introduction of RZV would be reduced by $8,600,33,000$, and 71,000 , respectively. This would be associated with 111,000 fewer HZ-related PCP visits and 650 fewer HZ-related hospitalizations over 15 years. The estimated incremental budget impact for the With RZV scenario compared with the Without RZV scenario would be $\$ 96$ million, \$158 million, and $\$ 180$ million (\$0.39, \$0.33, and \$0.25 average incremental PMPM among all plan members) over 5,10 , and 15 years, respectively.

\section{SENSITIVITY ANALYSIS}

Among the key model inputs for which alternate assumptions were explored for the commercial, Medicare, and KPSC health plans, cumulative incremental case avoidance and budget impact over 10 years from RZV adoption were generally most sensitive to maximum RZV coverage levels achieved (Supplementary Figure 1, available in online article). HZ-related costs and second dose compliance for RZV had relatively less effect on budget impact over 10 years post-RZV implementation than vaccine coverage for the commercial and KPSC health plans. Despite this, incremental budget impact projections for these plans varied by as much as $33 \%$ when assumptions around costs and second dose compliance were varied. Varying the costs per case of HZ had a greater effect on the Medicare plan than on the commercial and KPSC plans. Data for sensitivity analyses at 5 and 15 years are available in Supplementary Figures 2 and 3 (available in online article). 


\section{Discussion}

\section{KEY FINDINGS}

In this study, we explored the clinical and economic impact of introducing RZV across varying health plan populations, time horizons, and with alternative key model assumptions. Analyses predicted that after the introduction of RZV, the projected increase in $\mathrm{HZ}$ vaccine coverage among those with and without previous HZ vaccination would lead to increases in HZ cases avoided and associated cost offsets over time. Compared with a continuation with ZVL, the increased efficacy and duration of protection afforded by RZV contributed to greater $\mathrm{HZ}$ case avoidance through both primary vaccination and repeat vaccination of those previously vaccinated with ZVL.

The estimated incremental PMPM budget impact of introducing RZV decreased over time after RZV adoption, as the incremental PMPM vaccine-related costs plateaued or decreased while incremental PMPM HZ-related cost savings increased. In essence, the shorter-term costs of adopting the new vaccine technology were increasingly offset by the longer-term benefits in the form of HZ case avoidance.

The Medicare health plan had proportionally more adults eligible for vaccination than the commercial health plan, resulting in additional RZV vaccination costs. However, as older adults are at a higher risk of HZ, more cases and complications of $\mathrm{HZ}$ were avoided and the HZ-related costs were reduced by a larger amount, resulting in a lower incremental budget impact compared with commercial health plans. The use of patient copays for each dose of RZV also reduced incremental vaccination costs for the Medicare health plan.

Sensitivity analyses demonstrated that uncertainty in coverage would generally have the greatest effect on budget impact, followed by uncertainty in second dose compliance. It should be noted that the effect of second dose compliance depends upon time horizon due to higher efficacy and less waning for 2 doses of RZV; the longer the time horizon the larger the effect of compliance on case avoidance. The cost per case uncertainty has a larger influence on incremental budget impact in plans with older individuals (e.g., Medicare plans) than in plans with younger individuals (e.g., commercial plans), as older individuals are at increased risk for complicated HZ cases.

\section{VALUE OF PLAN-SPECIFIC INFORMATION}

This analysis demonstrates the flexibility of the model to provide plan-specific information to PBDMs considering adoption of RZV. In the KPSC case study using epidemiological data specific to a single IDN, a similar incremental HZ case avoidance was observed over 15 years compared with the hypothetical commercial plan (28.1\% of HZ cases avoided vs. $29.6 \%$, respectively). Estimates of annual HZ incidence for the KPSC population were similar to national estimates. ${ }^{15,33}$ However, the magnitude of HZ-related PCP visits and hospitalizations avoided differed from the hypothetical commercial health plan (even after adjusting for differences in population size), as the health care resource utilization estimates per $\mathrm{HZ}$ case were lower for the KPSC population, ${ }^{15}$ than for the national population, ${ }^{25}$ possibly as a result of different methodologies to determine these inputs. However, since national estimates were used to estimate the costs for managing $\mathrm{HZ}$ cases in the KPSC scenario, PMPM budget impact for KPSC tracked closely to the hypothetical commercial health plan over time.

\section{MODEL VALIDITY AND TRANSPARENCY}

This model analysis had several strengths. A third party, the KPSC study team, was involved in model adaptation, consistent with recommendations for pharmacoeconomic models. ${ }^{34}$ The inputs, calculations, and assumptions in the model are transparent, allowing the possibility for independent assessment of face validity of the structure, input data, and results from the model. In addition, feedback obtained via payer interviews was incorporated to ensure the relevance of the model outcomes. Internal verification exercises were also conducted, including structured walkthroughs of the model code, rigorous reviews of each piece of the model's calculations, and extreme condition tests for model input values.

As the model is being made publicly available (Supplementary File 1, available in online article) and has a user-friendly interface, it can be customized by payers and PBDMs to tailor to their own plans/needs. Plan-specific data use when available for model inputs will maximize the value of the model outcomes for decision makers. Furthermore, as was recently suggested in a study on BI model validation, ${ }^{35}$ it could be expected that with planspecific data, the predictive validity of the BI model could be confirmed once real-world outcomes for RZV vaccination emerge in the coming years.

\section{LIMITATIONS}

While clinical trials demonstrated RZV to be efficacious in the prevention of HZ, the vaccine's characteristics-efficacy, waning, second dose compliance-may vary in the real world. Of note, the efficacy of RZV was estimated in a healthy immunocompetent population with no history of HZ. As real-world effectiveness data become available, the effectiveness of RZV in people with a history of HZ, against recurrent $\mathrm{HZ}$ after breakthrough cases, and in immunocompromised individuals will become clearer, as will the duration of protection. 
Real-world vaccine coverage could differ from that forecast in this modeling exercise. This would affect the budget impact of RZV, as demonstrated in sensitivity analyses.

In the KPSC case study, national cost estimates were applied for the cases avoided as plan-specific data for HZ-related costs were not available. Health plans would be able to generate more relevant plan-specific budget impact estimates by using their own cost-per-HZ-case data. Additionally, changes in health plan demographics (e.g., members leaving or joining) could affect outcomes from the model.

\section{Conclusions}

Model projections demonstrate that RZV adoption allows the reduction of $\mathrm{HZ}$ cases and complications. Results for representative national populations with Medicare or commercial insurance coverage show that the projected health gains would increase and that incremental PMPM costs would decrease over longer time horizons. The BI model (Supplementary File 1, available in online article) can be adapted to support plan-specific decision making related to RZV introduction.

\section{DISCLOSURES}

GlaxoSmithKline Biologicals SA funded this study (GSK study identifier: HO-1718378) and was involved in all stages of study conduct, including analysis of the data. GlaxoSmithKline Biologicals SA also paid all costs associated with the development and publication of this manuscript.

Patterson, Van Oorschot, and Curran are employees of the GSK group of companies and hold shares in the GSK group of companies. Herring, Carrico, and Zhang are employees of RTI Health Solutions, which received funding via a contractual agreement with the GSK group of companies to perform the work contributing to this research. Ackerson, Bruxvoort, Sy, and Tseng are employees of Kaiser Permanente Southern California, which was contracted by the GSK group of companies for the conduct of this study and were members of the KPSC study team. Ackerson, Bruxvoort, Sy, and Tseng report research contracts with the following pharmaceutical companies unrelated to this study: Dynavax (Ackerson, Bruxvoort, and Sy); the GSK group of companies (Ackerson, Bruxvoort, Sy, and Tseng); Novavax (Ackerson, Sy, and Tseng); and Seqirus (Ackerson, Bruxvoort, Sy, and Tseng). Tseng reports having served as a paid consultant for the GSK group of companies. The authors declare no other financial and nonfinancial relationships and activities.

Findings from this study were presented at AMCP Nexus 2019; October 29November 1, 2019; National Harbor, MD.

\section{ACKNOWLEDGMENTS}

The authors thank the additional members of the KPSC study team-Yun Tian, Yi Luo, Hilary Tanenbaum, Chengyi Zheng, and Bianca Cheung-for providing scientific and administrative support in collection and analysis of KPSC epidemiological data, as well as David Wamble from RTI Health Solutions for providing technical input and gathering data about payer preferences for utilizing budget impact models. Also, the authors thank Business \& Decision Life Sciences platform for editorial assistance and manuscript coordination, on behalf of GSK. Grégory Leroux coordinated manuscript development and editorial support. The authors also thank Rachel Emerson (Business \& Decision Life Sciences, on behalf of GSK) for providing medical writing support.

\section{REFERENCES}

1. Oxman MN. Herpes zoster pathogenesis and cell-mediated immunity and immunosenescence. J Am Osteopath Assoc. 2009;109(6 Suppl 2):S13-S17.

2. Yawn BP, Wollan PC, St Sauver JL, Butterfield LC. Herpes zoster eye complications: rates and trends. Mayo Clin Proc. 2013;88(6):562-70.

3. Ozawa S, Portnoy A, Getaneh $\mathrm{H}$, et al. Modeling the economic burden of adult vaccine-preventable diseases in the United States. Health Aff (Millwood). 2016;35(11):2124-32.

4. McLaughlin JM, McGinnis JJ, Tan L, Mercatante A, Fortuna J. Estimated human and economic burden of four major adult vaccine-preventable diseases in the United States, 2013. J Prim Prev. 2015;36(4):259-73.

5. Varghese L, Standaert B, Olivieri A, Curran D. The temporal impact of aging on the burden of herpes zoster. BMC Geriatr. 2017;17(1):30.

6. Harpaz R, Ortega-Sanchez IR, Seward JF. Prevention of herpes zoster: recommendations of the Advisory Committee on Immunization Practices (ACIP). MMWR Recomm Rep. 2008;57(RR-5):1-30.

7. Dooling KL, Guo A, Patel M, et al. Recommendations of the Advisory Committee on Immunization Practices for use of herpes zoster vaccines. MMWR Morb Mortal Wkly Rep. 2018;67(3):103-08.

8. Morrison VA, Johnson GR, Schmader KE, et al. Long-term persistence of zoster vaccine efficacy. Clin Infect Dis. 2015;60 (6):900-09.

9. Tseng HF, Harpaz R, Luo Y, et al. Declining effectiveness of herpes zoster vaccine in adults aged $\geq 60$ years. $J$ Infect Dis. 2016;213(12):1872-75.

10. Cunningham AL, Lal H, Kovac M, et al. Efficacy of the herpes zoster subunit vaccine in adults 70 years of age or older. $\mathrm{N}$ Engl J Med. 2016;375(11):1019-32.

11. Lal H, Cunningham AL, Godeaux O, et al. Efficacy of an adjuvanted herpes zoster subunit vaccine in older adults. $\mathrm{N}$ Engl $\mathrm{J}$ Med. 2015;372(22):2087-96.

12. Mauskopf J, Earnshaw S. A methodological review of US budget-impact models for new drugs. Pharmacoeconomics. 2016;34(11):1111-31.

13. Graham J, Mauskopf J, Kawai K, Johnson KD, Xu R, Acosta CJ. Budgetimpact analysis of alternative herpes zoster vaccine strategies: a U.S HMO perspective. J Manag Care Spec Pharm. 2016;22(7):872-88. doi: 10.18553/ jmcp.2016.22.7.872

14. Arias E, Heron M, Xu J. United States life tables, 2012. Natl Vital Stat Rep. 2016;65(8):1-65. Accessed September 8, 2020. https://www.cdc.gov/nchs/data/ nvsr/nvsr65/nvsr65 08.pdf

15. Tseng HF, Bruxvoort K, Ackerson B, et al. The epidemiology of herpes zoster in 
immunocompetent, unvaccinated adults $\geq 50$ years old: incidence, complications, hospitalization, mortality, and recurrence. J Infect Dis. 2020;222(5):798-806.

16. Curran D, Patterson B, Varghese L, et al. Cost-effectiveness of an adjuvanted recombinant zoster vaccine in older adults in the United States. Vaccine. 2018;36(33):5037-45.

17. Curran D, Patterson BJ, Van Oorschot D, et al. Cost-effectiveness of an adjuvanted recombinant zoster vaccine in older adults in the United States who have been previously vaccinated with zoster vaccine live. Hum Vaccin Immunother. 2019;15(4):765-71.

18. Curran D, Van Oorschot D, Varghese L, et al. Assessment of the potential public health impact of herpes zoster vaccination in Germany. Hum Vaccin Immunother. 2017;13(10):2213-21.

19. Centers for Disease Control and Prevention. Archived CDC vaccine price list as of September 1, 2019. 2019. Accessed September 8, 2020. https:// www.cdc.gov/vaccines/programs/vfc/ awardees/vaccine-management/pricelist/2019/2019-09-01.html

20. Centers for Disease Control and Prevention. Archived CDC vaccine price list as of June 1, 2018. 2018. Accessed September 8, 2020. https://www.cdc. gov/vaccines/programs/vfc/awardees/vaccine-management/pricelist/2018/2018-06-01.html

21. Ortega-Sanchez I. Decision and costeffectiveness analyses of herpes zoster vaccination in adults 50 years of age and older. National Center for Immunization and Respiratory Diseases, Centers for Disease Control and Prevention. October 23, 2013. Accessed September 8, 2020. https://stacks.cdc.gov/view/cdc/75337
22. The Office of the Legislative Counsel. Compilation of Patient Protection and Affordable Care Act. 111th Congress, 2nd Session. June 9, 2010. Accessed September 8, 2020. https://www.hhs.gov/sites/ default/files/ppacacon.pdf

23. U.S. Bureau of Labor Statistics. Consumer Consumer Price Index. All urban consumers, medical care. Generated interactively. 2019. Accessed September 8, 2020. https://www.bls.gov/cpi/

24. Johnson BH, Palmer L, Gatwood J, Lenhart G, Kawai K, Acosta CJ. Healthcare resource utilization and costs associated with herpes zoster in the US. J Med Econ. 2016;19(10):928-35.

25. Yawn BP, Itzler RF, Wollan PC, Pellissier JM, Sy LS, Saddier P. Health care utilization and cost burden of herpes zoster in a community population. Mayo Clin Proc. 2009;84(9):787-94.

26. Centers for Disease Control and Prevention. Vaccination coverage among adults in the United States, National Health Interview Survey, 2015. 2015. Accessed September 8, 2020. https:// www.cdc.gov/vaccines/imz-managers/ coverage/adultvaxview/coverage-estimates/2015.html

27. Dooling K. Herpes zoster vaccines update. In: Advisory Committee on Immunization Practices (ACIP). Summary report. February 27-28, 2019. Pages 149-51. Accessed September 22, 2020. https:// www.cdc.gov/vaccines/acip/meetings/ downloads/min-archive/min-2019-02508.pdf

28. Barnett JC, Vornovitsky MS. Health insurance coverage in the United States: 2015. September 2016. Accessed September 8, 2020. https://www.census. gov/content/dam/Census/library/publications/2016/demo/p60-257.pdf
29. Centers for Medicare \& Medicaid Services. 2014 Medicare enrollment section. 2014. Accessed September 8, 2020. https://www.cms.gov/ Research-Statistics-Data-and-Systems/

Statistics-Trends-and-Reports/ CMSProgramStatistics/2014/2014 Enrollment

30. Nelson JC, Bittner RCL, Bounds L, et al. Compliance with multiple-dose vaccine schedules among older children, adolescents, and adults: results from a vaccine safety datalink study. Am J Public Health. 2009;99(Suppl 2):S389-97.

31. Meyers JL, Madhwani S, Rausch D, Candrilli SD, Krishnarajah G, Yan S. Analysis of real-world health care costs among immunocompetent patients aged 50 years or older with herpes zoster in the United States. Hum Vaccin Immunother. 2017;13(8):1861-72.

32. Sullivan SD, Mauskopf JA, Augustovski F, et al. Budget impact analysis-principles of good practice: report of the ISPOR 2012 Budget Impact Analysis Good Practice II Task Force. Value Health. 2014;17(1):5-14.

33. Yawn BP, Saddier P, Wollan PC, St Sauver JL, Kurland MJ, Sy LS. A population-based study of the incidence and complication rates of herpes zoster before zoster vaccine introduction. Mayo Clin Proc. 2007;82(11):1341-49.

34. Olson BM, Armstrong EP, Grizzle AJ, Nichter MA. Industry's perception of presenting pharmacoeconomic models to managed care organizations. J Manag Care Pharm. 2003;9(2):159-67. doi: 10.18553/jmcp.2003.9.2.159

35. Hung A, Slejko JF, Lugo A, Shaya F, Haines ST, Mullins CD. Validating a budget impact model using payer insight and claims data: a framework and case study. J Manag Care Spec Pharm. 2019;25(8):91321. doi: 10.18553/jmcp.2019.25.8.913 